\title{
Modeling Optimal Scheduling for Pumping System to Minimize Operation Cost and Enhance Operation Reliability
}

\author{
Yin Luo, Shouqi Yuan, Yue Tang, Jianping Yuan, \\ and Jinfeng Zhang \\ Research Center of Fluid Machinery Engineering and Technology, Jiangsu University, \\ Zhenjiang 212013, China \\ Correspondence should be addressed to Yin Luo, luoyin6@gmail.com
}

Received 20 October 2011; Revised 18 January 2012; Accepted 22 January 2012

Academic Editor: Zhiwei Gao

Copyright (c) 2012 Yin Luo et al. This is an open access article distributed under the Creative Commons Attribution License, which permits unrestricted use, distribution, and reproduction in any medium, provided the original work is properly cited.

Traditional pump scheduling models neglect the operation reliability which directly relates with the unscheduled maintenance cost and the wear cost during the operation. Just for this, based on the assumption that the vibration directly relates with the operation reliability and the degree of wear, it could express the operation reliability as the normalization of the vibration level. The characteristic of the vibration with the operation point was studied, it could be concluded that idealized flow versus vibration plot should be a distinct bathtub shape. There is a narrow sweet spot (80 to 100 percent BEP) to obtain low vibration levels in this shape, and the vibration also follows similar law with the square of the rotation speed without resonance phenomena. Then, the operation reliability could be modeled as the function of the capacity and rotation speed of the pump and add this function to the traditional model to form the new. And contrast with the tradition method, the result shown that the new model could fix the result produced by the traditional, make the pump operate in low vibration, then the operation reliability could increase and the maintenance cost could decrease.

\section{Introduction}

As important aspects in engineering industries, low cost and high reliability are the focus of the operation control in pumping system $[1,2]$.

The purpose of pump scheduling function is to schedule the operation of $N$ pumps over a time period to meet consumer demands, and optimizing this function has been proven to be a practical and highly effective method in reducing operation costs without altering the actual infrastructure of the whole system. Thus, this issue naturally draws the attention of researchers [2]. 
Pump system scheduling should be robust with any operation scenarios and should deal virtually with all operation factors, such as variable speeds, constant speeds, and switched-off pumps, in relation with operation constraints relative to power, head, flow, and speed. It is a very complex problem.

Many researchers have developed optimal control concepts to minimize operating costs associated with water-supply pumping systems. Mays [3] listed and classified various algorithms that have been developed to solve the associated control problem. In earlier studies, linear, nonlinear, integer, dynamic, mixed, and other kinds of programming were used to optimize a single objective: reduction of the electric energy cost. A detailed review of these works can also be found in [4]. Later, Lansey et al. [5] introduced the number of pump switches as an alternative to evaluate the pumps' maintenance costs, which became the second objective considered until that time. This method also proved that one Gulf Coast refinery project [6] can save about $\$ 2$ million per year. In this way, as far as pump operation is concerned, the basic optimal scheduling model to reduce electrical and maintenance costs was formulated. The development of the pump scheduling model was later modified by forming or adding some optimization objectives, such as reservoir level variation and power peak, according to specific conditions.

However, in contrast to the estimation of electrical cost, where the computation is straightforward, computing for maintenance costs using the number of pump switches has some limitations.

According to some researchers [7-10], the cost of unscheduled maintenance which was not considered in former model may be the largest contributor to operation cost in process plants, and although with the mainly aspect of the wear and tear in switch course, the operation course should not been ignored.

At the same time, Bloch and Geitner [7] published three hydraulic factors, namely, rotation speed, impeller diameter (tip clearance), and operation point that can affect operation reliability which affects the unscheduled maintenance directly.

Subsequently, further improvements can be realized if these hydraulic factors could be quantified to develop an objective model in pump scheduling to enable the pump to operate in a high-reliability mode, then the reliability incidents would be reduced. As a result, maintenance cost can be reduced [10].

Vibration is one of the most vexing problems of pumping machineries, and it is the primary cause of considerable altercations and litigations. Excessive vibration of pumps and piping can destroy parts of the equipment (such as drive shafts, bearings, and seals). It can affect the reliability and life of the equipment and is often assumed as direct sign of reliability and health of the machine. One major end user puts a great deal of emphasis in reducing pump vibration through precise maintenance program, and some researchers adopted this index to indicate the degree of health and reliability of pumps. For example, Perez proposed a new method based on vibration called Nelson plot to assess the risk of low-flow operation in centrifugal pumps [11].

The main purpose of the current study is to set the vibration level as quantification of the hydraulic factors that influence operation reliability and the wear and tear in operation. Based on analysis of the vibration characteristics, an objective model is formulated to describe the relative reliability during operation, which presents a quantitative approach to evaluate alternative operating conditions. This new objective is added to the traditional model to form a new scheduling model. Subsequently, the operating conditions of the pump can be improved by making the pump operate at low vibration, the maintenance cost will be reduced, and the operation reliability will be enhanced to a certain degree. 


\section{Vibration Characteristics of Pumps}

For centrifugal pumps, the sources or causes of vibration are mainly composed of two types: mechanical and hydraulic.

For the mechanical cause, some imbalance and shaft misalignment always exist. This can cause vibration of the pump, and the intensity of this kind of vibration is related to the excitation force caused by the imbalance of the load. The load is directly related to the operation point.

Hydraulic vibration is caused by the reaction of the impeller vane as it passes the casing cutwater. Pump operation offers the best efficiency point (BEP) and thus creates eddies within the pump and some flow instabilities, such as cavitation. All these factors directly correlate with the operation point.

As throttle and speed controls are the most common control methods in centrifugal pumping system, the vibration characteristics under these two control operating modes were analyzed.

\subsection{Vibration Characteristics of the Pump under Throttle Control}

Basically, three types of vibrations should be distinguished: free vibrations, forced vibrations, and self-excited vibrations. Whereas free vibrations are rarely significant in pump operations, forced and self-excited vibrations frequently cause problems. For the forced vibrations, the force is the cause of the vibration, and self-excited vibration occurs when the exciting frequency is close to the natural frequency; it is an abnormal condition that should be avoided in system design and installation. Therefore, the vibration characteristics can be obtained by studying the mechanical and hydraulic force characteristics.

A force curve is shown in Figure 1 [12], summarized by Vorhoeven through test results from 47 pumps. The horizontal axis represents the BEP multiples, and the vertical axis represents the dimensionless number of the forces, expressed as

$$
K_{R}=\frac{F}{\rho g H D_{2} b_{2}}
$$

where $F$ is the force, $\rho$ is the fluid density, $H$ is the pump head, $D_{2}$ is the outer diameter of the impeller, and $b_{2}$ is the outlet width of the blade.

From the curve, the force caused by imbalance has a remarkable characteristic: it does not become small because of the BEP, and it is related to the load. When the load exceeds the $\mathrm{BEP}$, the force significantly increases, and this force is relatively stable and small when the pump operates with partial load.

For the force generated by the flow, some features can be found. The minimum excitation is in the range of $0.9 \mathrm{BEP}$ to $\mathrm{BEP}$, and the excitation increases with partial load and at high flow condition. These curves are displayed in distinct bathtub shape. The stronger excitation caused by the interaction has a large slope at the right of the BEP, whereas the other has smaller slope at the left of the BEP.

From the above discussion, conclusion can be drawn that the idealized flow versus vibration plot should have a distinctive bathtub shape. For this bathtub shape, a narrow sweet spot $(80 \%$ to $100 \%$ BEP) exists that can be used to obtain low vibration levels. The 


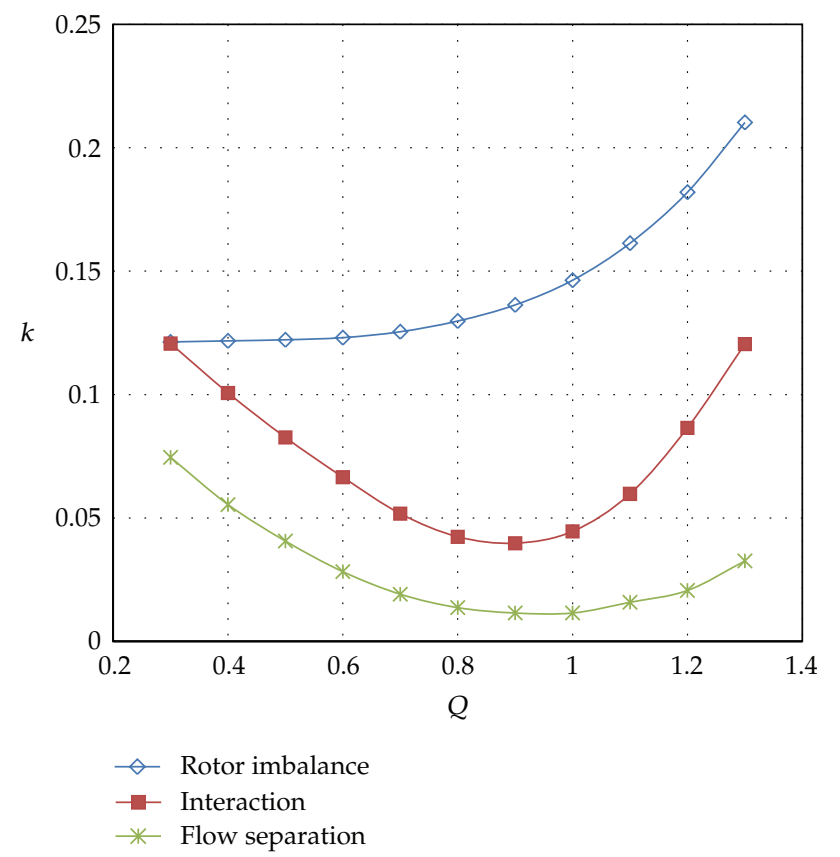

Figure 1: Forces in the impeller.

capacities move to the left and right of the low vibration level point, the vibration levels begin to increase, and the slope at the right area is larger than that at the left.

This bathtub-shaped flow versus vibration plot also can be found in some research works [9-11].

Figure 2 shows the test result of a well-designed centrifugal pump obtained by $\mathrm{Ni}$ et al. [13]. The pump was precision manufactured and installed. In Figure 2, BX, BY, and BZ are three direction vibration levels of the bearing house, whereas pc is the vibration level of the pump cover. The flow versus vibration plot in that test is exactly as the expression, and similar result can also be found in Perez's work [11].

\subsection{Vibration Characteristics of the Pump under Speed Control}

Mechanical and hydraulic excitations are the main causes of vibration.

According to the affinity law in pumps, when a pump is operating at two different speeds, the flow condition in the pump is homologous. Thus, when the pump operates at different rotation speeds, the variable tendency of hydraulic excitation versus the flow is still the same as that at different speeds, but the degree of some flow phenomena, such as instability interaction, will change under different speeds, especially at low speed.

The mechanical excitation is mostly related to the balance degree and the load. The load still has some homologous regularities expressed as the variable tendency of hydraulic excitation versus flow, which is almost the same at different speeds. In addition, once the speed drops below the nominal motor speed, the mechanical excitation is reduced quite obviously. 


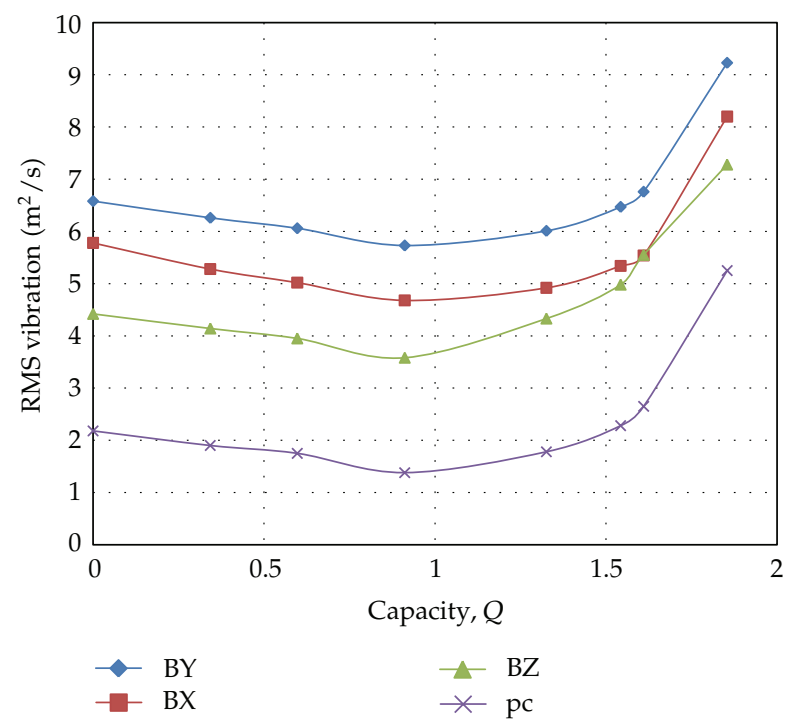

Figure 2: Vibration level in a well-designed centrifugal pump by Ni.

Based on the above results, the excitation versus flow at different rotor speeds is noted to have similar varying tendency to a certain extent, but the degree of excitation is different. Therefore, the vibrations among different rotor speeds from this point have similar varying tendency to a certain extent.

Figure 3 [10] shows the test vibration level dates of a pump in a chemical process with model 1.5 $\times 3$-13 ANSI B 73.1. The measured point of vibration is at the thrust bearing horizontal plane, and the date was adopted with root mean square (RMS) expression. The vibrations among different rotor speeds have similar varying tendency to a certain extent.

However, in centrifugal pumps, the vibration spectrum usually contains only the fundamental shaft frequency, the blade passage frequency, and one or two harmonics. All these characteristic frequencies are directly decided by the rotor speed. Therefore, potential dangers of resonance exist, and when resonance occurs at the characteristic frequency close to the natural frequency, the similar varying tendencies are gone. Thus, in practice, lockout speed range is recommended.

As all forces are proportional to the product of pressure $\times$ area, $F \sim n^{2} \times d^{4}$ also applies.

For a given pump, the hydraulic excitation forces increase with the square of the tip speed and the density, that is, $\rho \times u^{2}$. The bearing housing and shaft vibrations of the pump would also increase with $\rho \times u^{2}$ if it were not for resonance phenomena.

In Stavale's work [10], the overall vibration was measured for a variable-speed test and compared with a constant-speed system with throttle valve, as shown in Figure 4. During the variable-speed test, the system was fixed, that is, there was no throttling of the control valve to initiate changes in the flow. Prior to the variable-speed test, the control valve was opened wide, and the backpressure valve was adjusted to obtain maximum flow at maximum speed; the backpressure was approximately zero.

Figure 4 shows that in the course of the variable-speed operation, the pump can operate by following the same operating condition line for the approximately zero backpressure. The vibration level of the square of the flow under the same operating 


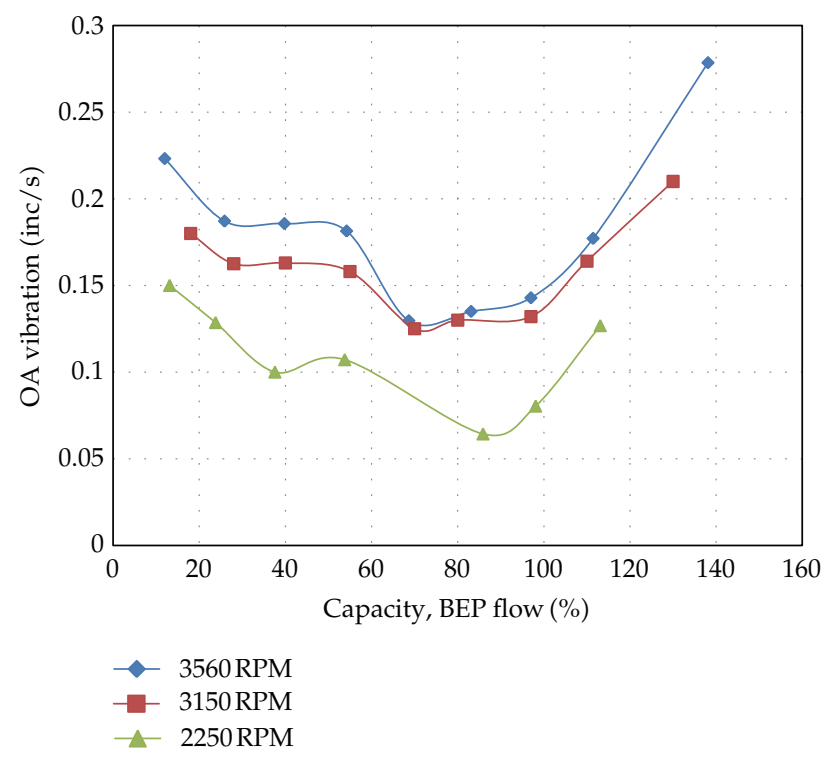

Figure 3: Test vibration level dates in different pump obtained by Stavale.

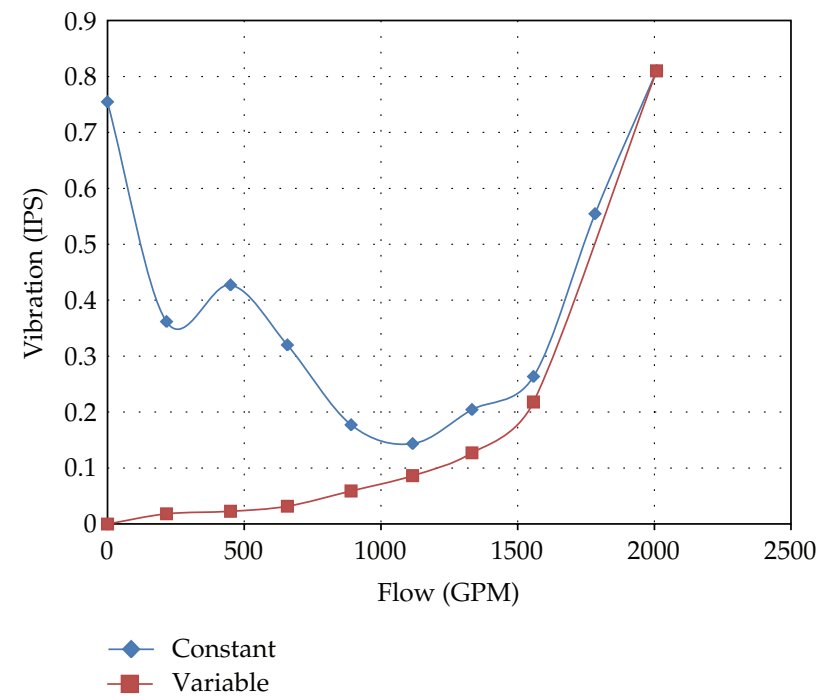

Figure 4: Vibration levels for different operating models of the same pump.

condition line can be clearly seen, so the vibration also follows the same law for the resonance phenomena of a given pump $V_{e} \sim n^{2}$, expressed as

$$
\frac{V_{e}\left(n_{1}\right)}{V_{e}\left(n_{2}\right)}=\frac{n_{1}^{2}}{n_{2}^{2}}
$$

where $n_{1}$ and $n_{2}$ represent two different operating pump speeds. 


\section{Maintenance Cost Model in Pump Operation}

Maintenance costs cannot be easily estimated, however, the wear of pumps is mainly caused by frequent switching them on and off. Formally, a pump switch is defined as turning on a pump which was previously off. Therefore, minimizing the number of pump switches will result in minimization of maintenance costs. As a result, the traditional method assumes that it increases as the number of "pump switched" increases, and its model can be built according to Lansey and Awumah [5] and Vladimir et al. [14].

In many applications, the cost of unscheduled maintenance which was not considered in former model may be the largest contributor to operation cost in process plants, and although with the mainly aspect of the wear in switch course, the operation course should not been ignored.

The life of the mechanical seal is directly related to shaft movement. Vibration can cause carbon face chipping and seal face opening. Drive lugs will wear, and metal bellows seals will fatigue. In some instances, the shaft movement can cause the rotating seal components to contact the inside of the stuffing box, or some other stationary object, causing the seal faces to open and allowing solids to penetrate between the lapped faces. Vibration is also a major cause of set screws becoming loose and slipping on the shaft, causing the lapped seal faces to open. The vibration would also cause denting of the bearing races for no design for the bearings to handle both a radial and axial load.

Critical dimensions and tolerances such as wear ring clearance and impeller setting will be affected by vibration.

Bearing seals are very sensitive to shaft radial movement. Shaft damage will increase and the seals will fail prematurely. Labyrinth seals operate with a very close tolerance. Excessive movement can damage these tolerances also.

Vibration can affect the reliability and life of the equipment and is often assumed to be the direct as direct sign of the reliability and health of the machine.

As vibration can affect the reliability and life of the equipment, indicators must be set up to consider vibration data. Maintenance cost is further assumed to increase as the reliability indicators reduce. In addition, maintenance cost can be significantly reduced with operation in a high-reliability mode, thus reducing wear and reliability incidents.

\subsection{Indicators of Operation Reliability}

Vibration data can be normalized based on the following equation:

$$
R=\left(1-\left(\frac{V}{V_{\max }}\right)\right)+C
$$

where $V$ is the vibration level at a certain point, $V_{\max }$ is the maximum date value, $C$ is a constant added to set the peak value of $R$ (equal to one) equal to $V_{\min } / V_{\max }$, and $R$ is the relative reliability indicator.

The $R$ value in (3.1) is a relative reliability number between zero and one. A zero value does not necessarily indicate zero reliability but is rather intended to discourage running the pump at these conditions. Similarly, a value of one does not indicate infinite reliability but is intended to be a relative indicator of the best operating conditions for a given pump. As the mechanical design of a pump can also affect reliability, these values should not be used to 
compare pumps of different designs or manufacturers. It is intended to compare the effect of alternative operating conditions on reliability.

\subsection{Maintenance Cost Model during Operation Based on Reliability Indicators}

\subsubsection{Single-Pump Model}

For a fixed-speed pump, the mathematical model can be expressed as a set of static relationships between the vibration level and flow rate. The pump vibration level is referred to as vibration velocity or vibration displacement, expressed as RMS or peak-to-peak values. The vibration level data are divided by the lowest of all the flows for simplification and normalization. The flow rate is also referred to as capability. Then, a single model can be expressed as a cubic or fourth-order polynomial equation for the bathtub-shaped curve:

$$
V=\bar{v}_{0}+\bar{v}_{1} Q+\bar{v}_{2} Q^{2}+\bar{v}_{3} Q^{3}
$$

where $V$ is the normalized vibration level at a certain point, $Q$ is the flow rate, and system parameters $v_{\mathrm{i}}$ are determined by specific pump vibration characteristics and can be identified by test data.

With regard to the variable-speed pump, the relationship between (3.2) and its parameters is motor-speed dependent. The affinity law in pump theory states the following:

$$
\frac{Q\left(n_{1}\right)}{Q\left(n_{2}\right)}=\frac{n_{1}}{n_{2}}, \quad \frac{H\left(n_{1}\right)}{H\left(n_{2}\right)}=\frac{n_{1}^{2}}{n_{2}^{2}}, \quad \frac{P\left(n_{1}\right)}{P\left(n_{2}\right)}=\frac{n_{1}^{3}}{n_{2}{ }^{3}},
$$

where $n_{1}$ and $n_{2}$ represent two different operating pump speeds.

Assuming that the pump model (3.2) for a VSD at a special speed $n_{0}$ is obtained and its indicators are $v_{0}, v_{1}, v_{2}$, and $v_{3}$, the pump model of the considered VSD for a given limited speed $n$ has the property defined by (2.2) and (3.2), that is,

$$
\begin{aligned}
\frac{V(n)}{V\left(n_{0}\right)} & =\frac{V(n)}{v_{0}+v_{1} Q\left(n_{0}\right)+v_{2} Q\left(n_{0}\right)^{2}+v_{3} Q\left(n_{0}\right)^{3}}=\frac{n^{2}}{n_{0}^{2}}, \\
V(n) & =\frac{n^{2}}{n_{0}^{2}}\left[v_{0}+v_{1} Q\left(n_{0}\right)+v_{2} Q\left(n_{0}\right)^{2}+v_{3} Q\left(n_{0}\right)^{3}\right] .
\end{aligned}
$$

Then, according to (3.3), with $k=n / n_{0}$, this part can be transformed into

$$
\begin{aligned}
V(n) & =\frac{n^{2}}{n_{0}^{2}}\left[v_{0}+v_{1}\left(Q(n) * \frac{n_{0}}{n_{1}}\right)+v_{2}\left(Q(n) * \frac{n_{0}}{n_{1}}\right)^{2}+v_{3}\left(Q(n) * \frac{n_{0}}{n_{1}}\right)^{3}\right] \\
& =k^{2} v_{0}+k v_{1} Q(n)+v_{2} Q^{2}(n)+k^{-1} v_{3} Q^{3}(n) .
\end{aligned}
$$


Subsequently, according to (3.1), the operation reliability of the signal pump can be expressed as

$$
R_{\mathrm{sig}}=\left(1-\left(\frac{V(n, Q)}{V_{\max }}\right)\right)+\frac{V_{\min }}{V_{\max }}
$$

$R_{\text {sig }}$ is a function of $Q$ (flow rate) and $n$ (rotation speed). It is directly related to the operating conditions and reflects the relationship between the maintenance cost and the operating conditions. The higher $R_{\text {sig }}$ is, the lower is the maintenance cost in the course of operation.

\subsubsection{MultiPump Model}

The general multiple pump systems consist of some special characteristics for operating maintenance cost analysis.

(1) The model should only reflect the pumps that are in operation. Pumps that are not running should not be considered.

(2) As maintenance cost is not the same for different pumps, the model should consider the difference.

(3) The relative normalization number is also between zero and one, similar to the single-pump model.

Considering the above-mentioned factors, the following methods are adopted.

(a) A swift variable vector is added to represent the operating condition of the pump group. Then, only the pump in operation is considered; hence, the program (2.1) can be solved.

(b) As the cost of pump is usually related to the installed capacity, assumption is made that the bigger the installed capacity is, the higher is the maintenance cost. Thus, a weight constructed with the ratio of single installed capacity to total installed capacity is adopted to represent the different maintenance costs.

From the above discussion, conclusion is made that the maintenance cost model for multipump system with $k$ pumps can be expressed as follows:

$$
R=\sum_{i=1}^{k} \frac{w_{i} \varphi_{i} R_{\text {sigi }}}{w_{i} \varphi_{i}}
$$

where

$$
w_{i} \in\{0,1\}, \quad i=1,2 \ldots k
$$

This equation is the swift variable vector. " 1 " shows that the pump is working, and " 0 " indicates that the pump is off:

$$
\varphi_{i}=\frac{N_{d i}}{N_{d T}}, \quad i=1,2 \ldots k
$$




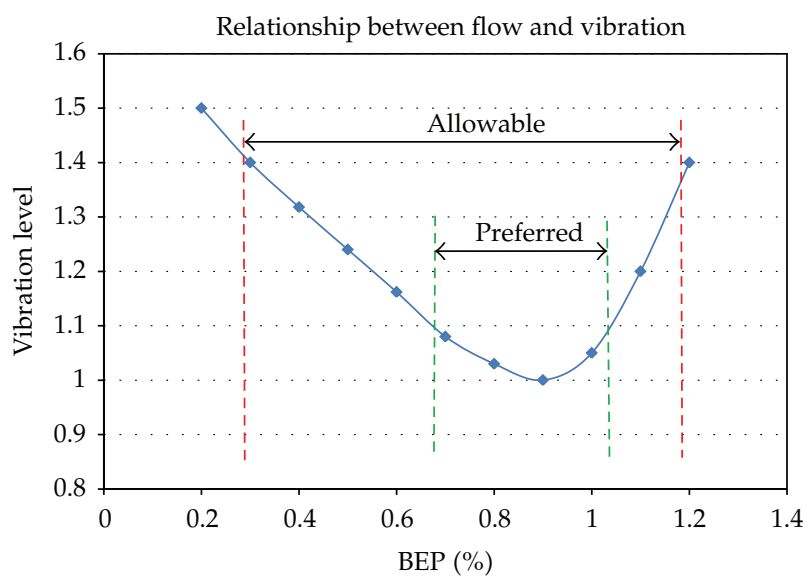

Figure 5: Idealized flow versus vibration plot recommended by API 610.

$N_{d T}$ is the total installed capacity of the multipump system, and $N_{d i}$ is single installed capacity of the $i$ th pump.

\subsubsection{Modeling Method of the Maintenance Cost without Test Data}

As environment and reliability requirements increase, the pumps are built and tested to standards and specifications that define the maximum allowable vibration amplitudes (process pumps and almost all large pumps) in many applications. However, unlike the pump characteristics, some installations still do not have such vibration data because no mandatory standard is required. Thus, the maintenance cost model may not work. Hence, other modeling methods must be developed to deal with this situation.

Fortunately, although specific vibration data may not be known, the allowable vibration amplitudes defined in standards, such as API 610 [15], are clear. The standards define different vibration limits for a "preferred operation range" and an "allowed operation range." In the preferred operation range, the maximum increment of vibration is less than $10 \%$. On the other hand, the maximum increment of vibration is less than $30 \%$ for the allowed operation range. In a certain pumping system, if the preferred and the allowed operation ranges are known, assumption can be made that all systems meet the standard. Therefore, a maintenance cost model can be developed.

Even if the preferred and allowed operation ranges are unknown, API 610 still provides an idealized flow versus vibration plot [11], as shown in Figure 5. Maintenance cost model can also be developed based on the assumption that the pump follows this law.

\section{Optimization Model}

Generally, the optimal policy should result in the lowest total operating cost for a given set of boundary and system constraints. Thus, objective function and constraints are needed for optimal scheduling model. 


\subsection{Objective Function}

In a typical pumping system, the operation cost mostly comprises energy-consumption cost and maintenance cost. Thus, the objective function also comprises these two factors [16-19].

\subsubsection{Energy Cost}

Pump scheduling is used for dealing with the following two situations.

One is the selection process on which available pumps are to be used and for what period of time (e.g., a day) the pumps should operate.

The other is referred to as (real-time) control problem. The optimal strategy is concerned with which available pumps must be operated and when they should be operated according to fluctuations in demands and/or operating conditions.

For the first situation [14], the objective is the determination of energy consumption of all pumps in the pumping station during the optimization period. The charging structure used by the electric utility is the factor that must be considered in analyzing electric energy cost. Then, the objective function is mathematically expressed as

$$
E=\sum_{i=1}^{I} \sum_{t=1}^{T}\left(E R_{i t}\right) C Q_{i t}\left(H S_{i t}\right),
$$

where $E$ is the total energy cost to be minimized, $I$ is number of pump systems, and $T$ is number of time intervals that constitute the operating horizon. $E R_{i t}$ is the electrical rating of pump $i$ during time period $t, C$ is the conversion coefficient, and $Q_{i t}$ and $H S_{i t}$ are the discharge and pressure head, respectively, of pump $i$ during time period $t$.

For the second situation [16], the real scheme is to consider the total input electrical power as the objective function. This method is much closer to the real situation. However, the calculation time will be long, which may not be suitable for real-time control. Thus, the modeling method commonly used is by considering the shaft horsepower of the pumps as objective function because the energy consumption of the motor and the inverter is very much less than that of the pump. Then, the objective function can be mathematically expressed as

$$
f 1=\sum_{i=1}^{I} w_{i} P_{i}\left(Q_{i}, k_{i}\right)
$$

where $f 1$ is the total shaft horsepower to be minimized, $I$ is number of pumps, and $Q_{i}$ and $k_{i}$ are the discharge and pressure head, respectively, of pump $i$ under certain operating conditions. $P_{i}$ is the shaft horsepower of pump $i$ under this condition, which can be expressed as (3.9) according to the characteristic of the pump and the affinity law, and $p_{i}$ is the conversion coefficient:

$$
P(Q, k)=p_{0} k^{3}+p_{1} k^{2} Q+p_{2} k Q^{2}+p_{3} Q^{3} .
$$




\subsubsection{Pump Maintenance Cost}

The pump maintenance cost can be as important as the electric energy cost or even more relevant; however, this cost cannot be quantified easily, but it can be described through other correlative factors indirectly. Thus, the number of "pump switched" and the operation relative reliability indicators are chosen to describe this factor.

\section{(1) The Number of "Pump Switched"}

Due to the difficulty in starting-up the pumps and the significant increase in frequency during their switching, the maintenance cost increases as the number of "pump switches" increases, which can be expressed as follows when real control is used for the pump schedule:

$$
d_{H}\left(w, w^{\prime}\right)=\sum_{i=1}^{n}\left|w_{i}-w_{i}^{\prime}\right|
$$

where $I$ is the number of pumps, $w_{i}$ is the operating status of pump $i$ for the next step, $w_{i}^{\prime}$ is the present operating status, $w_{i}$ and $w_{i}^{\prime}$ are both switch variables, 1 indicates that the pump is in operation, and 0 shows that the pump is off.When the pump schedule is used for this scheme for a period of time, the objective function becomes the total switch number at this time, expressed as

$$
N_{s}=\sum_{t=1}^{T} \sum_{i=1}^{n}\left|w_{i}^{t}-w_{i}^{t-1}\right|
$$

\section{(2) Operation Relative Reliability Indicators}

$R$ in (3.6) and (3.7) is a relative reliability number between zero and one. As $R$ approaches one, the higher the reliability is, the lower is the pump wear and the longer is the MTBR; subsequently, maintenance cost will be reduced.For the real-time control, (3.7) can be used directly to express the scheme in a period of time. The summation of the time periods can be used as the objective function.

\subsection{Constraints}

\subsubsection{Water-Supply Demand Constraints}

For the pump system to meet some of the water-supply requirements [17], the desired watersupply index, which is known for the pump system, can be expressed as $\left(H_{\mathrm{ST}}, Q_{e}\right)$, expressed in a mathematic model.

For parallel-connected pump systems,

$$
\begin{gathered}
Q_{e}=\sum_{i=1}^{n} Q_{i}, \\
H_{\mathrm{ST}}=H_{i} .
\end{gathered}
$$


For series-connected pump systems,

$$
\begin{gathered}
Q_{e}=Q_{i}, \\
H_{\mathrm{ST}}=\sum_{i=1}^{n} H_{i} .
\end{gathered}
$$

\subsubsection{Operation Constraints}

Obviously, a pump should be selected so that it operates predominantly close to the BEP in the so-called "preferred operation range." This mode of operation is apt to produce the lowest energy and maintenance costs and minimize the risk of system problems. However, off-design operation for limited periods cannot be avoided. Rules are needed to define the allowable ranges and modes of operation to reduce the risk of damage and excessive wear. To this effect, limits must be defined for continuous and for short-term operations at maximum and minimum flow [16].

The range of preferred continuous operation can be defined, for instance, by the requirement that the efficiency must not fall below $80 \%$ to $85 \%$ of the maximum efficiency of the pump, and allowable ranges can be defined so that the efficiency must not fall below $70 \%$.

This constraint can be expressed as

$$
\begin{aligned}
\min \left(Q_{\mathrm{POP} i}\right) & \leq Q_{i} \leq \max \left(Q_{\mathrm{POP} i}\right) \\
\min \left(Q_{\mathrm{AOP} i}\right) & \leq Q_{i} \leq \max \left(Q_{\mathrm{AOP} i}\right) .
\end{aligned}
$$

For optimal operation, the constraint should be in POP, as shown in (4.9). However, for some situations, the optimization model may have no solution. In this case, the constraint is adjusted to AOP; then, (4.10) can be applied.

At the same time, when the pump is operated by VSD, the speed regulation range should also be constrained. The operation efficiency will decrease because of a very wide speed range, and reliability will reduce. Therefore, the factors for operation should be considered. The speed regulation range is constrained as $\left[k_{\min } 1\right] \cdot k_{\min }$ is determined by several factors, such as the rotation speed at which the pump is no longer able to maintain discharge against the static head from the demand side, the rotation speed to avoid system resonance, and the rotation speed to ensure that the pump operating economically.

As the pump operates under speed-control model, the range of the continuous and short-term operations will constantly change.

According to the affinity law in pump theory, $l_{1}$ and $l_{2}$ (Figure 6) are set as similar lines, and $\mathrm{A}, \mathrm{B}, \mathrm{C}$, and D are the boundaries of the operation zone; then, $l_{1}$ and $l_{2}$ can be expressed as

$$
H_{l 1}=\frac{H_{A}}{Q_{A}} Q_{l 1}{ }^{2}, \quad H_{l 2}=\frac{H_{B}}{Q_{B}} Q_{l 2}{ }^{2} .
$$




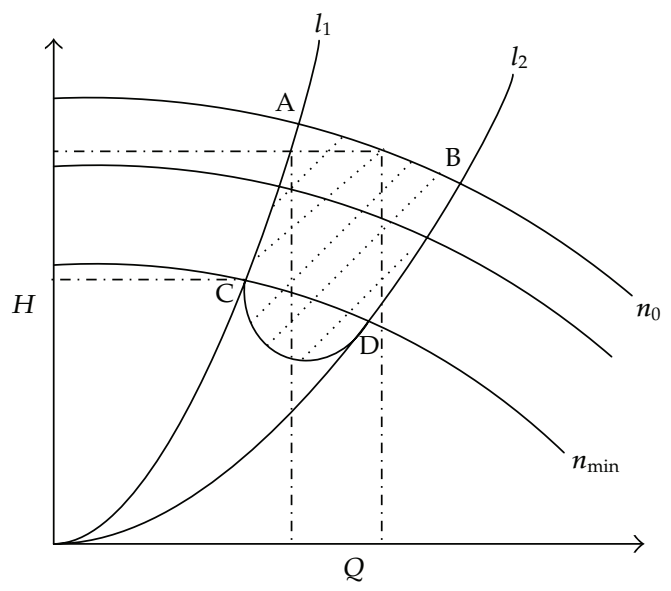

Figure 6: Efficient operation area for the variable-speed pump. changed:

If head $H_{e}$ is needed for this water supply system, then the boundary could be

$$
\begin{gathered}
Q_{\min }= \begin{cases}Q_{A} \sqrt{\frac{H_{e}}{H_{A}}} & H_{e} \geq H_{C}, \\
Q_{c} & H_{e}<H_{C},\end{cases} \\
Q_{\max }= \begin{cases}Q_{B} \sqrt{\frac{H_{e}}{H_{B}}} & H_{e}<H_{B}, \\
Q_{B} & H_{e} \geq H_{B} .\end{cases}
\end{gathered}
$$

\section{Application}

\subsection{Profiles of the Pump Station}

A sample model is a circulating water pumping station, one of the most important facilities in an alumina plant used for the mother liquor evaporation process. It uses almost $17 \%$ of the electricity consumption in the plant, and the reliability of this system is very important for the whole plant.

There are five pumps of single- and double-stage suction in the pumping system, and the pump model is shown in Table 1.

Variable-speed control is adopted for $1^{\#}$ and $2^{\#}$, and the minimum speed regulation ranges $\left(k_{\min }\right)$ are 0.7 and 0.75 in this system.

The operation characteristics of these pumps are shown in Table 2.

For this pumping system, the basic demand characteristic obeys (4.10) and can be calculated based on process requirements and pipeline characteristics.

$$
H_{\mathrm{dem}}=42+3.51 \times 10^{-7} Q^{2} .
$$


Table 1: The configuration and the parameters of the pump.

\begin{tabular}{lcccccc}
\hline Serial number & Pump model & $Q_{\text {den }}(\mathrm{m} 3 / \mathrm{h})$ & $H_{\text {den }}(\mathrm{m})$ & $Q_{\min }(\mathrm{m} 3 / \mathrm{h})$ & $Q_{\max }(\mathrm{m} 3 / \mathrm{h})$ & $N(\mathrm{RPM})$ \\
\hline $1^{\#}$ & $14 S H-9 B$ & 1425 & 58 & 855 & 1853 & 1450 \\
$2^{\#}$ & $20 S A-10$ & 2850 & 58 & 1710 & 3848 & 960 \\
$3^{\#}$ & $20 S A-10$ & 2850 & 58 & 1710 & 3848 & 960 \\
$4^{\#}$ & $20 S A-10$ & 2850 & 58 & 1710 & 3848 & 960 \\
$5^{\#}$ & 20SA-10 & 2850 & 58 & 1710 & 3848 & 960 \\
\hline
\end{tabular}

Table 2: The model parameters of the pump.

\begin{tabular}{|c|c|c|c|c|}
\hline \multicolumn{5}{|c|}{$H=H x-S Q^{2}$} \\
\hline Serial number & $H_{x i}$ & $S_{i}$ & & \\
\hline $1^{\#}$ & $71.17 k_{1}^{2}$ & $7.488 e-6$ & & \\
\hline $2^{\#}$ & $70.39 k_{2}^{2}$ & $1.780 e-6$ & & \\
\hline $3^{\#}$ & 70.39 & $1.780 \mathrm{e}-6$ & & \\
\hline $4^{\#}$ & 70.39 & $1.780 e-6$ & & \\
\hline $5^{\#}$ & 70.39 & $1.780 e-6$ & & \\
\hline \multicolumn{5}{|c|}{$P=P_{0}+P_{1} Q+P_{2} Q^{2}+P_{3} Q^{3}$} \\
\hline Serial number & $P_{0}$ & $P_{1}$ & $P_{2}$ & $P_{3}$ \\
\hline $1^{\#}$ & $146.4 k_{1}^{3}$ & $0.05 k_{1}^{2}$ & $4.4 e-5 k_{1}$ & $-1.44 e-8$ \\
\hline $2^{\#}$ & $230.5 k_{2}^{3}$ & $0.1025 k_{2}^{2}$ & $5.826 e-6 k_{2}$ & $-2.1 e-9$ \\
\hline $3^{\#}$ & 230.5 & 0.1025 & $5.826 e-6$ & $-2.1 e-9$ \\
\hline $4^{\#}$ & 230.5 & 0.1025 & $5.826 e-6$ & $-2.1 e-9$ \\
\hline $5^{\#}$ & 230.5 & 0.1025 & $5.826 e-6$ & $-2.1 e-9$ \\
\hline \multicolumn{5}{|c|}{$V=V_{0}+V_{1} Q+V_{2} Q^{2}+V_{3} Q^{3}$} \\
\hline Serial number & $V_{0}$ & $V_{1}$ & $V_{2}$ & $V_{3}$ \\
\hline $1^{\#}$ & $7.696 e-10 k_{1}^{2}$ & $-1.57 e-6 k_{1}$ & $3.84 e-4$ & $1.483 k_{1}^{-1}$ \\
\hline $2^{\#}$ & $9.62 e-11 k_{2}^{2}$ & $-3.936 e-7 k_{2}$ & $1.92 e-4$ & $1.483 k_{2}^{-1}$ \\
\hline $3^{\#}$ & $9.62 e-11$ & $-3.936 e-7$ & $1.92 e-4$ & 1.483 \\
\hline $4^{\#}$ & $9.62 e-11$ & $-3.936 e-7$ & $1.92 e-4$ & 1.483 \\
\hline $5^{\#}$ & $9.62 e-11$ & $-3.936 e-7$ & $1.92 e-4$ & 1.483 \\
\hline
\end{tabular}

The whole flow date is shown in Figure 7, and these values are obtained from the water demand curve based on historical data.

\subsection{Optimization Pump Scheduling and the Result}

A one-day optimization result was made. Assumption was made that the shortest period for each combination of pumps is $1 \mathrm{~h}$, that is, a pump can be switched off/on after having been active or inactive for at least $1 \mathrm{~h}$.

Based on the flow demand in Figure 7 and the head demand in (4.10), the desired objective value is shown in Figure 8.

Genetic algorithm was selected as the optimization method because of its suitable characteristics for adaptability to complex optimization problem $[18,19]$. 


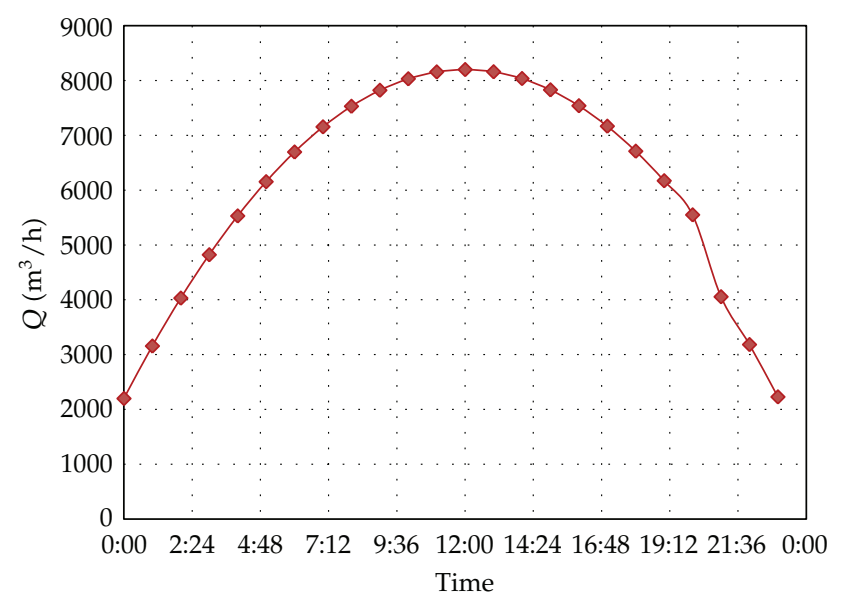

Figure 7: Daily variation curve of the entire flow demand.

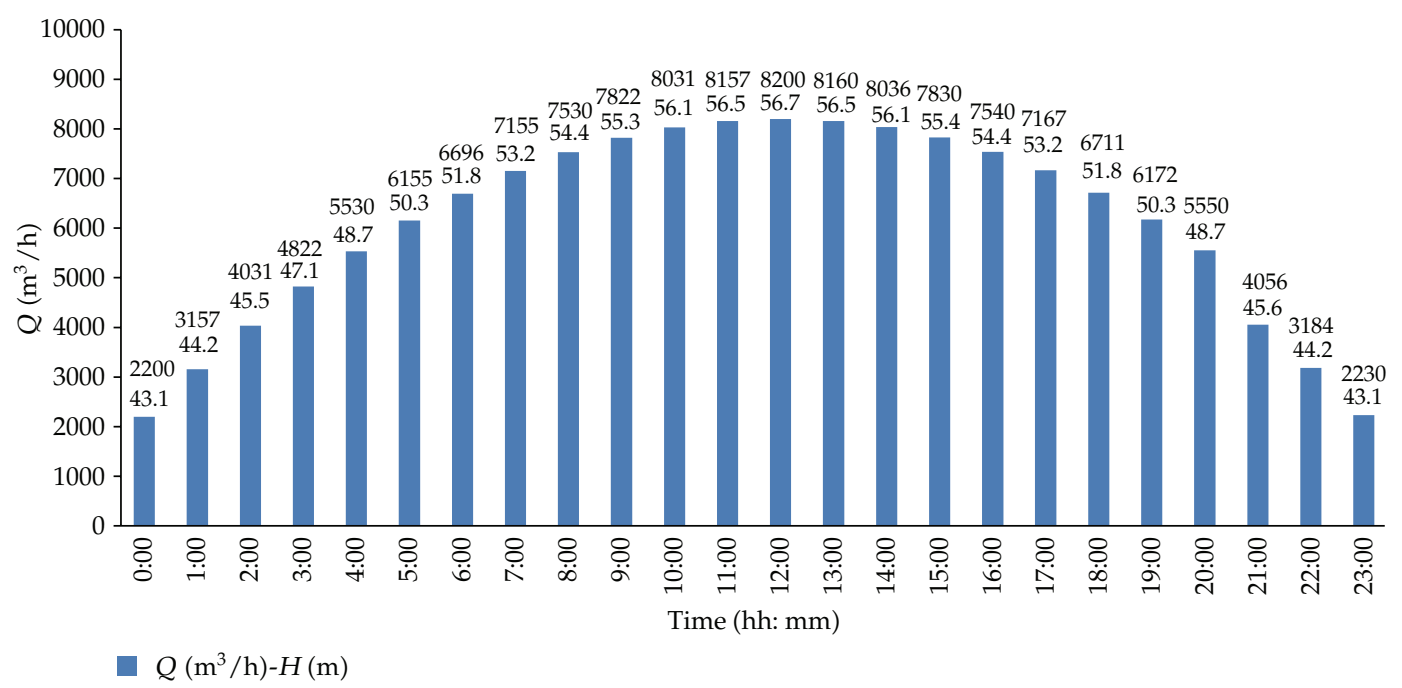

Figure 8: Daily supply index.

In this optimization problem, the single objective approach developed by Mackle was adopted because of its simplicity. The fitness function consists of the energy consumption cost and penalties for violation of the constraints of the system. All these factors were linearly weighted.

Then, the optimal result is shown in Figures 9, 10, and 11. A represents the result when only the minimum energy cost is considered, and B represents the result when both maintenance and energy costs are considered.

Figure 9 shows the result of the shaft power in the two different optimization objectives under the same water supply index. The result considered that the energy consumption was smaller, and the multiobjects are almost equal to the whole, whereas in some cases, they were smaller. This condition might be because the high efficiency point is the most reliable operation point in most situations; however, in some cases, the general shaft 


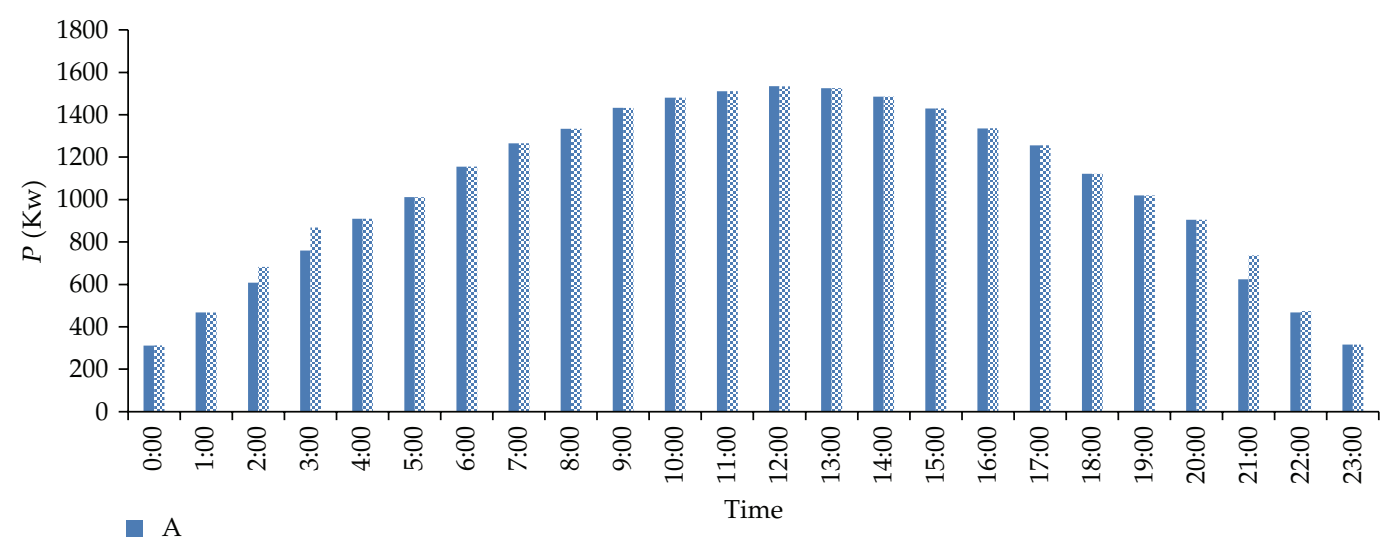

$\mathrm{A}$
$\mathrm{B}$

Figure 9: Input power of the different models.

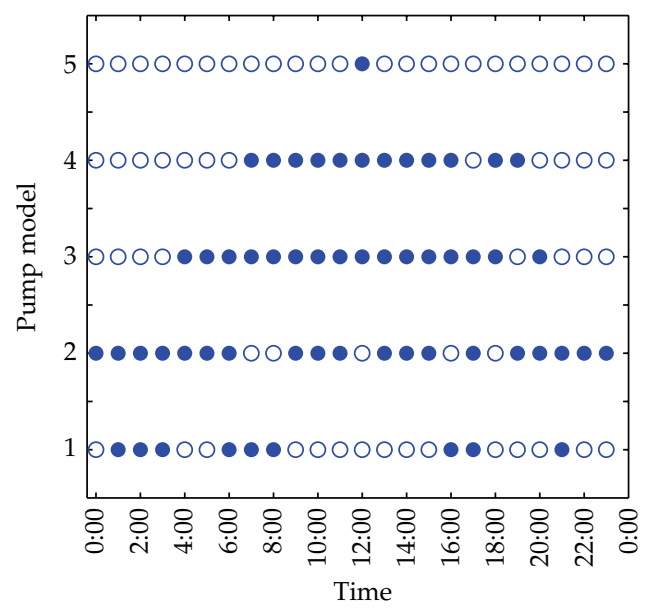

(a)

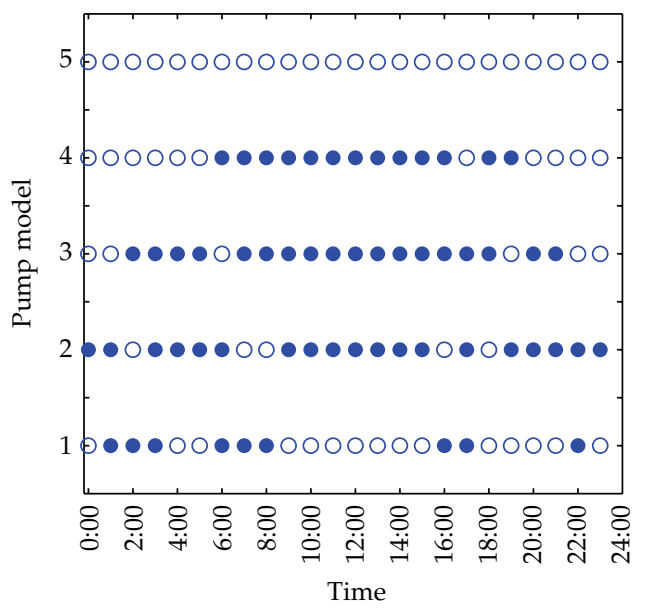

(b)

Figure 10: (a) Operation state of the form model. (b) Operation state of the new improved model.

power may be lower. For a single pump, operating in the high vibration point can cause the whole system to operate in unreliable conditions.

Thus, from energy-saving perspective, considering only energy cost as the main objective may be even better. However, when the switch number and reliability factor are considered in the optimization course, pump control are easy and simple using the least number of switches, and reliability will be enhanced when the operation point is close to the low vibration point as much as possible. Then, the pump system will be operating under high reliability condition, the maintenance cost will be reduced, and the service life will be extended. Subsequently, the whole LCC will be decreased by this kind of optimization scheduling model. 


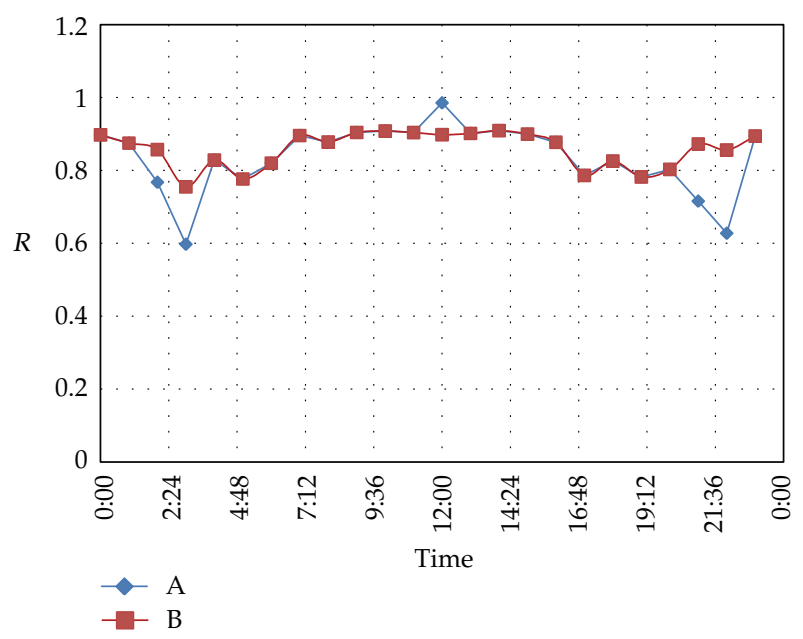

Figure 11: Reliability factor number of the different models.

\section{Conclusions}

In the present work, a new multiobjective approach, which takes into account operation reliability and maintenance cost incurred in operation, is presented.

The main conclusions from the current study are the following.

(1) Operation cost reduction and operation reliability enhancement are the focus of operation control for pump systems. Operation reliability for running pumps, which is a key to decrease unscheduled maintenance costs caused by the reliability incidents, and the wear cost during the operation should be considered.

(2) Vibration can affect the reliability and life of the equipment. An index reflecting the operation reliability and the wear degree in operation course could be considered as the normalization of vibration level.

(3) The idealized flow versus vibration plot should take a distinct bathtub shape. For this bathtub shape, a narrow sweet spot ( $80 \%$ to $100 \%$ BEP) exists that can be used to obtain low vibration levels, and the vibration also follows a similar law with the square of the rotation speed if it were not for resonance phenomena with a given pump.

(4) The maintenance cost which is not considered by traditional model concluding the unscheduled maintenance cost and the wear cost during the operation can be modeled as a function of the pump capacity and rotation speed based on the vibration characteristics. This function is then added to the traditional optimal scheduling model to create a new optimal scheduling model.

(5) Compared with the traditional method, the result of the new optimal model changes the result produced by the traditional one. It improves the operating conditions of the pump and enables the pump to run operate low vibration level. Therefore, maintenance cost can be reduced and the operation reliability can be enhanced to a certain degree.

\section{Acknowledgments}

This work is supported by National Outstanding Young Scientists Founds of China (Grant no. 50825902), Jiangsu Provincial Innovative Scholars “Climbing” Project of China (Grant no. 
BK 2009006), the National Natural Science Foundation of China (Grant no. 50979034), and Priority Academic Program Development of Jiangsu Higher Education Institutions.

\section{References}

[1] Hydraulic Institute, Pump Life Cycle Costs: A Guide to LCC Analysis for Pumping Systems, Parsippany, NJ, USA, 2001.

[2] Z.-H. Wang, G.-H. Geng, and S.-K. Song, "Discussion on energy conservation of pump," China Foreign Energy, vol. 11, no. 5, pp. 73-76, 2006 (Chinese).

[3] L. W. Mays, Water Distribution Systems Handbook, McGraw-Hill, New York, NY, USA, 2000.

[4] L. E. Ormsbee and K. E. Lansey, "Optimal control of water-supply pumping systems," Journal of Water Resources Planning \& Management, vol. 120, no. 2, pp. 237-252, 1994.

[5] K. E. Lansey and K. Awumah, "Optimal pump operations considering pump switches," Journal of Water Resources Planning and Management, vol. 120, no. 1, pp. 17-35, 1994.

[6] D. C. White, "Improve your project's prospects," Chemical Processing, vol. 67, no. 10, pp. 33-39, 2004.

[7] H. P. Bloch and F. K. Geitner, An Introduction Machinery Reliability Assessment, Gulf Publishing, Houston, Tex, USA, 1994.

[8] A. R. Budris, R. B. Erickson, F. H. Kludt, and C. Small, "Consider hydraulic factors to reduce pump downtime," Chemical Engineering, vol. 109, no. 1, pp. 54-60, 2002.

[9] R. B. Erickson, E. P. Sabini, and A. E. Stavale, Hydraulic Selection to Minimize the Unscheduled Maintenance Portion of Life Cycle Cost, Pump Users International Forum, Karlsruhe, Germany, 2000.

[10] A. E. Stavale, "Reducing reliability incidents and improving meantime between repair," in Proceedings of the 24th Interenational Pump Users Symposium, pp. 1-10, College Station, Tex, USA, 2008.

[11] X. Robert and P. E. Perez, "Operating Centrifugal Pumps Off-design- pumps \& systems 20 suggestions for a new analysis method operating centrifugal pumps," April 2005, http://www.pumpzone.com/articles/2.pdf.

[12] Y.-Y. Ni, 3-D unsteady numerical simulation and fluid-induced vibration for centrifugal pumps, Ph.D. thesis, Jiangsu University, ZhenJiang, China, 2008.

[13] Y.-Y. Ni, S.-Qi Yuan, Z.-Y. Pan et al., "Diagnosing the running condition of pump by its vibration character," Drainage and Irrigation Machinery, vol. 25, no. 2, pp. 49-52, 2007 (Chinese).

[14] C. Vladimir, M. Heiliö, N. Krejić, and M. Nedeljkov, "Mathematical model for efficient water flow management," Nonlinear Analysis, vol. 11, no. 3, pp. 1600-1612, 2010.

[15] API Standard 610, Centrifugal Pumps for Petroleum Heavy Duty Chemical and Gas Industry Services, Petrochemical and Natural Gas Industries, 9th edition, 2003.

[16] C. Zhang, H. Li, M. Zhong, and J. Cheng, "The modelling and optimal scheduling for pressure and flow varying parallel-connected pump systems," Dynamics of Continuous, Discrete and Impulsive Systems B, vol. 11, no. 6, pp. 757-770, 2004.

[17] T. C. Yu, T. Q. Zhang, and X. Li, "Optimal operation of water supply systems with tanks based on genetic algorithm," Journal of Zhejiang University, vol. 6, no. 8, pp. 886-893, 2005.

[18] B. Barán, C. Von Lücken, and A. Sotelo, "Multi-objective pump scheduling optimisation using evolutionary strategies," Advances in Engineering Software, vol. 36, no. 1, pp. 39-47, 2005.

[19] A. S. Dragan, A. W. Godfrey, and S. Martin, "Multiobjective genetic algorithms for pump scheduling in water supply," Lecture Notes in Computer Science: Evolutionary Computing, vol. 1305, pp. 227-223, 1997. 


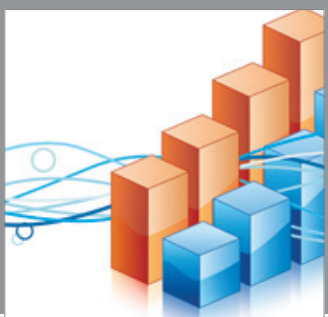

Advances in

Operations Research

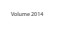

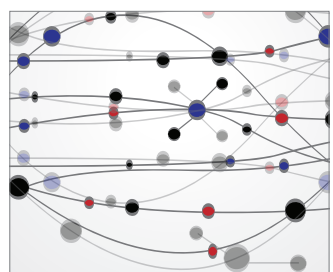

\section{The Scientific} World Journal
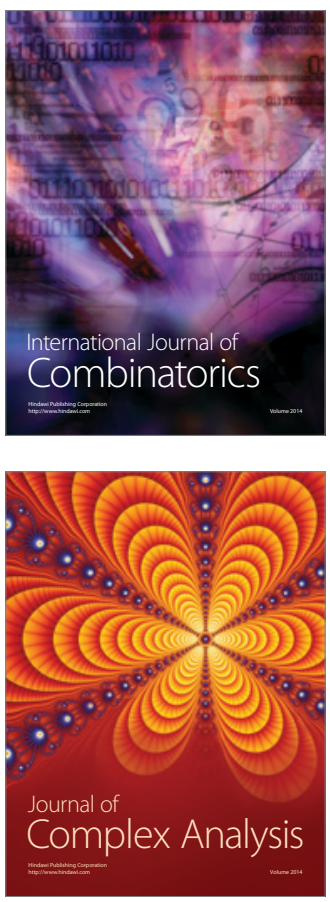

International Journal of

Mathematics and

Mathematical

Sciences
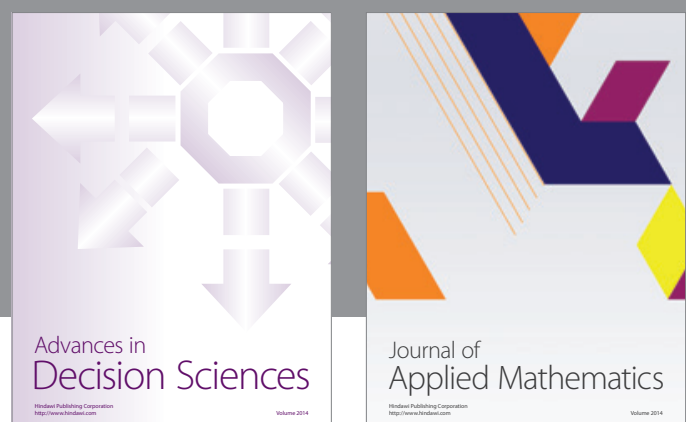

Journal of

Applied Mathematics
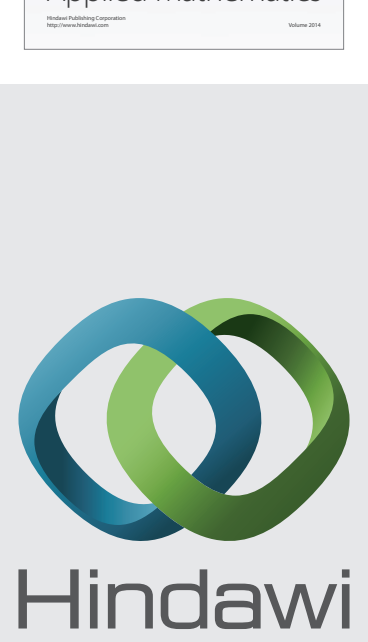

Submit your manuscripts at http://www.hindawi.com
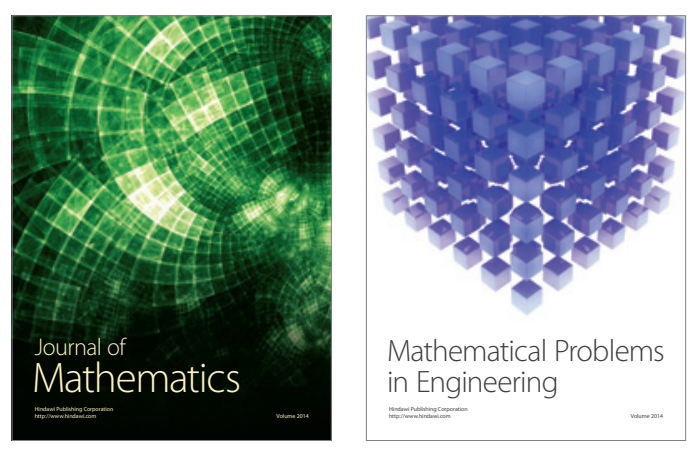

Mathematical Problems in Engineering
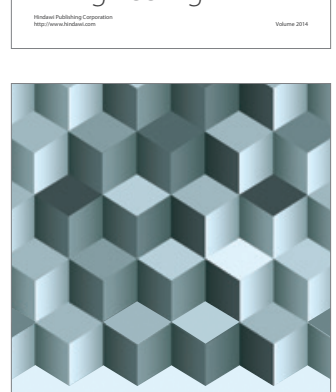

Journal of

Function Spaces
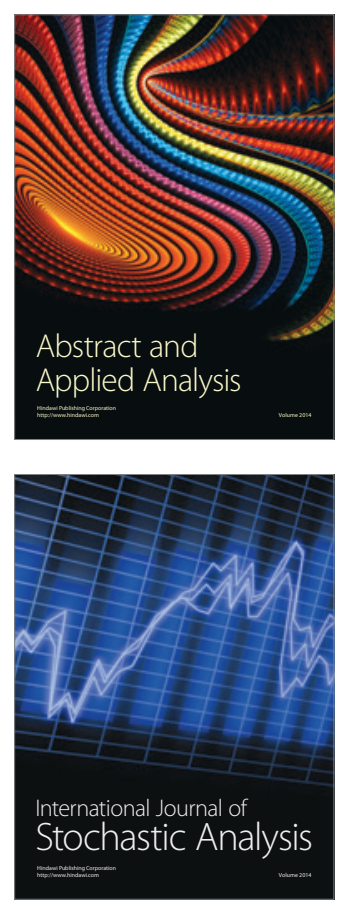

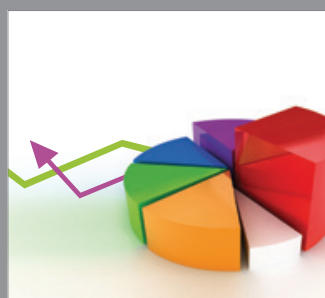

ournal of

Probability and Statistics

Promensencen
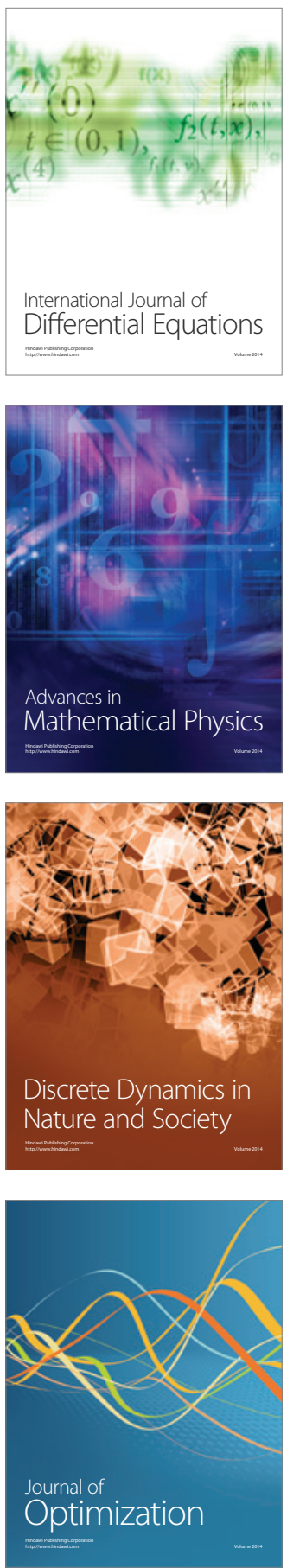